\title{
Application of Digital Mockup Technology
}

\author{
Gaoming Ding ${ }^{\mathrm{a}, *}$
}

${ }^{a}$ Jiandong College, CZJDU, Changzhou, China

\begin{abstract}
Digital simulation design is one of the mechanical product modern design methods. We have introduced the concept and meaning of digital mockup technology in mechanical design and its architecture, design cycle, which are concerned multi-domain UML, multi-body dynamics and multidisciplinary design. We also have analyzed the automobile digital simulation design method and digital mockup technology.
\end{abstract}

Index Terms: Digital simulation; Digital mockup; UML; Design optimization

(C) 2011 Published by MECS Publisher. Selection and/or peer review under responsibility of the Research Association of Modern Education and Computer Science

\section{Functional Virtual Prototyping}

With the development of virtual prototyping technology, computer simulation has been used in product design as checking and optimizing product design. We can test product model, simulate test and estimate product by means of digital analyzing technology. We can improve product efficiency and reduce cost when we improve design.

\subsection{Virtual prototyping}

Virtual prototyping technology is a kind of technology which used in simulation design process. It uses virtual prototyping to replace physical prototyping and tests the design about some or whole performance. Virtual prototype emphasizes the building of product's digital model and solving problems by means of all kinds of subjects.[1] It tests and improves the design of product by building a system or subsystem model which is similar to product physical machine to simulate which based on computer. So, we can acquire the optimized performance.

\subsection{Functional Digital Prototype}

Functional digital prototype is corresponding to the process of product analysis, especially the product's multi-performance analysis and multi-system dynamic performance optimization, which used to simulate the equipped system's whole function and operational performance. It is an expanded application about general

* Corresponding author:

E-mail address: hbxfqxyqxy_123@163.com 
virtual prototyping technology, and it fits the basic thoughts of product multi-subject design optimization. It pays attention to use CAD/CAE software system to integrate, which realizes the integration of product multifunction whole analysis. It is also used to solve such problems as dynamics, structure, strength and period which based on multi-object system and finite element theory. It forecasts the product system's whole performance precisely by means of virtual test.

\section{Multidisciplinary Design Optimization}

Multidisciplinary design optimization is a kind of method which designs complex system and subsystem by discovering and using coordinate systems in engineering system, these systems are interacted each other. Many subjects' design should be paid attention to notice the affection between products's different parts and the product's whole manufacture process.

\subsection{Complex product's MDO digitized flow}

Multidisciplinary design optimization must be mixed in complex mechanical product's digitized design flow and combined the existed digitized design method, advanced simulation technology, which forms a closed design process. This is helpful to design and improve design. So, we can get an automatic and integrated optimal design process.

Fig.1 shows the optimal flow about complex product's multidisciplinary design optimization which based on digitized prototyping. We can build the original digitized functional prototyping which based on product structure by designing multidisciplinary design synthetically. We also can use multidisciplinary modeling technology which guarded by main model to build component-order and subject-order analyzing models and getting the key technical parameters about product system's multi-performance optimization by means of CAD/CAE integrated software. We can analyze simulation result and design by means of the whole simulation and virtual testing. We can build digitized optimal model by means of model converting technology, which based on subject analysis model.

\subsection{FDP integrated design platform which based on $M D O$}

Functional virtual prototyping is the process of analyzing and simulating virtual model continuously in the product's whole period circle. Fig. 2 shows the FDP integrated design platform which based on MDO.

We can increase the ability of refining parameters and prescribing knowledge about digital model by introducing expert system. We choose object function in accordance with the performance evaluation system of functional virtual prototyping, and define different subjects' design requirements as optimal restriction. So, we can express the product's design purpose from the angle of multi-subject optimal design. We can integrate CAD/CAE software and multi-subject optimal methods by using methods which based on software interface, analyze and optimize product structure and performance by means of $\mathrm{CO}$ framework, check design by simulating the actual working state, fasten design process by means of optimization process' feedback. The whole process forms integrated automatic design simulation of functional virtual prototyping. 


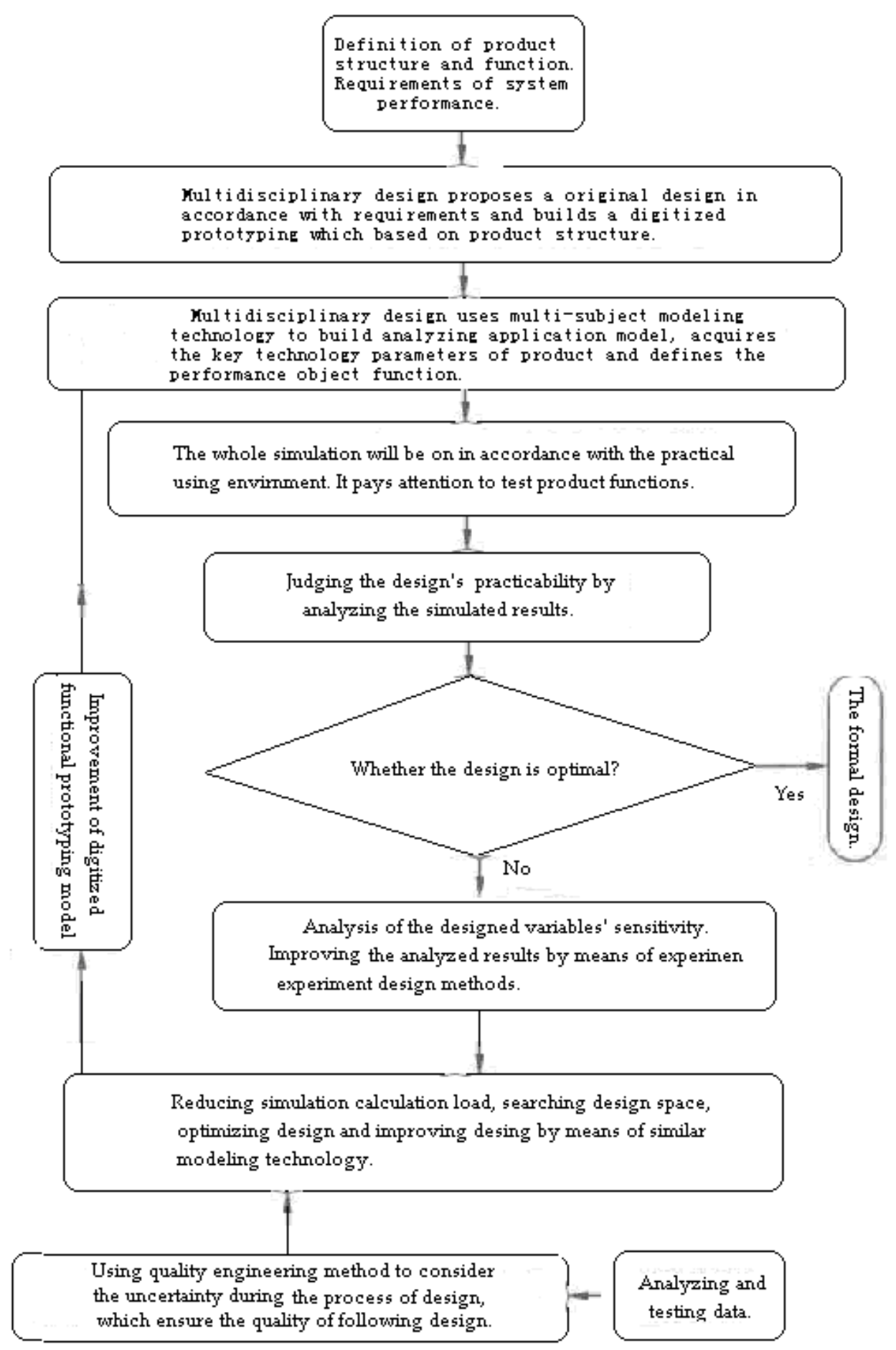

Fig. 1. Optimal flow of multidisciplinary design which based on digitized functional prototyping 


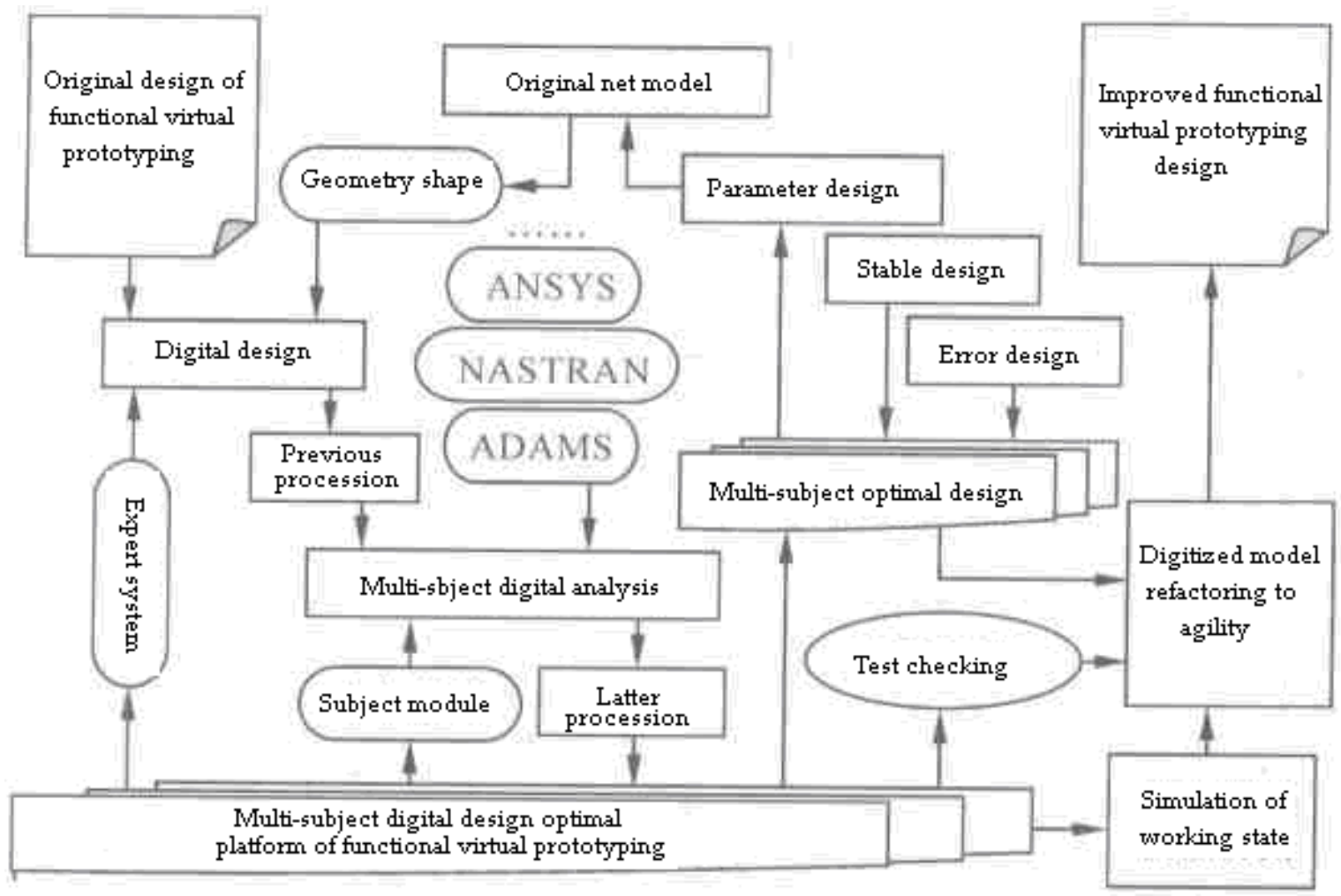

Fig. 2. FDP integrated design platform which based on MDO

\subsection{Integrated analysis simulation flow of FDP multi-subject}

According the whole design example of automobile, Fig.3 shows the integrated analysis and simulation flow about complex mechanical product's functional virtual prototyping. We can build automobile parameter structure model by means of Pro/E software which based on original design functional virtual prototyping, put parameter model signal into ADAMS by connecting MECHANISM/Pro with ADAMS software seamlessly, and simulate multi-system model by building CAE analyzing model. We can convert model under the environment of dual software by integrating ANSYS software into Pro/E seamlessly by means of ANSYS Utilities, and unify CAD/CAE really. We use LS-DYNA from ANSYS Corporation to simulate automobile collision directly. We analyze by combining PAM/CRASH software and ADAMS software which based on using ordinary data exchanging file format.[2]

We can conclude the design object after using ADMAS simulating software to integral analyzing. We also can get many key parameters about performance (object function) by analyzing and calculating the sensitiveness of subject parameters. So the product design can be improved greatly. 


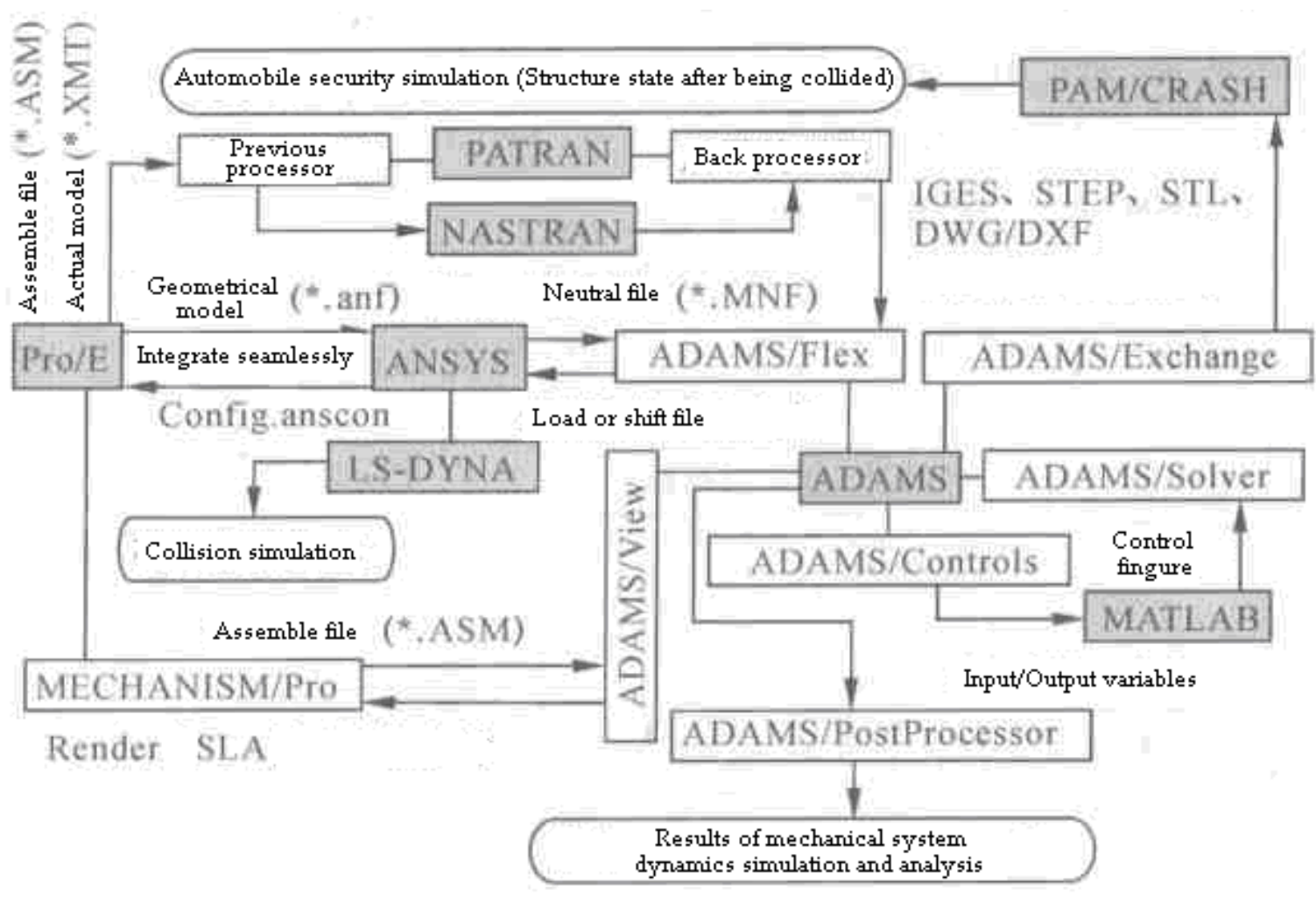

Fig. 3. Integrated analysis simulation flow of FDP multi-subject

Analysis is the basis of design. Functional virtual prototyping emphasizes the integration digital analysis of product performance and acquires the functional parameters by building model. The flow introduces the conversion between product structure main model and other subject analysis models by means of integration seamless of subject software. And we reduce the back processing time of the whole analyzing process by using finite element model and CAD/CAE software to sole many analyzing problems.[3] So the development circle of product can be shorten greatly.

\section{Application of Virtual Prototyping Technology in Automobile Engine Bonnet Lock}

Automobile engine bonnet lock is the auxiliary component of engine bonnet. Its main function is that it can ensure engine to lock safely. In the domain of automobile design, virtual prototyping technology is that using three-dimensional design software to build automobile model and simulate the model. Virtual prototyping builds a model which is similar with physical one by means of computer simulation technology. It prescribes the performance by optimizing, integrating and simulating. Virtual prototyping technology has been realized on the background of digital design. It depends on three-dimensional digital model's precision, and it can be used to simulate and analyze rapidly. So the design of product can be optimized greatly.

\subsection{Building bonnet lock model}

We first analyze the principle and structure of the existed automobile lock actual model. We need to measure the main components, and then build a three-dimensional model by means of UG software. A precise 
physical model and convenient modeling method is the key of virtual prototyping technology. During the process of modeling, we mainly need to use such functions as Sketch, Form Feature, Free Form Feature and Sheet Metal Feature.

\subsection{Building engine bonnet lock model}

The structure of automobile engine bonnet lock is very complex, and it is typical sheet-metal component. We use UG sheet-metal design module to get the model of engine bonnet lock. Sheet Metal Feature menu has such functions as General Flange, Sheet Metal Bend, Sheet Metal Punch, Sheet Metal Bead, Sheet Metal Bridge, and so on. These functions have been applied in the design of engine bonnet lock model widely. We can use UG software modeling method to build all components' actual model, which lays foundation for the back design and analysis. So the design quality can be improved greatly.[4]

\subsection{Virtual analysis of engine bonnet lock}

In order to study and analyze conveniently, we have built four designs: scenario1, scenario2, scenario3 and scenario4. Every kind of design is corresponding to a assemble array. We found many problems during the process of analyzing, such as the interval is bigger. We weren't satisfied until we had modified the virtual prototyping model timely. We have used virtual prototyping technology adequately. This has reduced the product developing circle and cost efficiently.

\subsection{Simulation of engine bonnet lock}

Motion module of UG has strong motion simulating and analyzing functions. It can reflect the application of virtual prototyping technology well. The whole process of motion simulation includes the following main steps: Creating link rod, Creating joints; Defining motion-driven, Creating motion-simulation.[5]

We have studied the motion way of bonnet lock in this paper. The engine bonnet moves from starting position to auxiliary hanger. At this time, the potential energy of engine bonnet will be converted into kinetic energy fully. So the lock can form the first lock-up by opening auxiliary hanger. But the kinetic energy of hanger will not be so strong as to move with bonnet. At this time, it needs people to put engine bonnet down. So the engine bonnet can be locked well.

In this paper, we have used joint motion method to simulate the motion of structure. The advantages of joint motion analysis include that we can use many motions at the same time without considering that every motion has same step number and step length.

\section{References}

[1] Li Bohu, Research and Practice on Complex Products Virtual Prototype Technology[J], MEASUREMENT \& CONTROL TECHNOLOGY, 2001 20(11)

[2] Li Ruitao, Conception and Application of Virtual Prototyping Technology[J], MECHATRONICS, 2000 $6(5)$

[3] Chen Gang, Specialized CAD/CAM System of Bevel Gear for Car Differential Mechanism based on UG[J], JOURNAL OF MECHANICAL TRANSMISSION, 2009 33(3)

[4] Lin Junmu, Dong Lin, UG-BASED REDEVELOPMENT OF CAD SYSTEM OF GEAR INJECTION MOLD[J], ENGINEERING PLASTICS APPLICATION, 2009 37(9)

[5] Zheng Quan, Chen Liqing, Development of Differential Gear CAD System Based on UG[J], JOURNAL OF MECHANICAL TRANSMISSION, 2009 33(1) 